\title{
Generation Means Analysis by Microcomputer
}

\author{
Timothy $\mathbf{J} \mathbf{N g}^{\mathbf{1}}$ \\ Department of Horticulture, University of Maryland, College Park, \\ MD 20742-5611
}

Additional index words. breeding, statistics, heritability, spreadsheet

Generation means tests are used by breeders and geneticists to assess additive, dominance, and epistatic variation in inheritance studies. Cavalli (1952) proposed a joint scaling test using the means of at least four families to determine the adequacy of an additivedominance model. This test involves estimating midparent $(\mathrm{m})$, additive ([d]), and dominance $([\mathrm{h}])$ parameters through a weighted least-squares analysis and testing the three-parameter model for goodness of fit. Failure of the model (m, [d], and [h]) is a definite indication of the presence of epistasis.

For crosses where epistasis is implicated, Jinks and Jones (1958) proposed a six-parameter scaling test to determine the adequacy of a digenic epistatic model. This test, which requires a minimum of six family means, estimates the $\mathrm{m}$, [d], and [h] parameters and also provides estimates of three epistatic parameters: homozygote $\times$ homozygote ([i]), homozygote $\times$ heterozygote (j]), and heterozygote $x$ heterozygote ([1]). The three-parameter and six-parameter scaling tests are described in detail by Mather and Jinks (1982).

Manual calculation of these scaling tests can be laborious and time-consuming. The advent of microcomputer technology and spreadsheet software allows these calculations to be performed rapidly and accurately, and without the use of an interpreted

Received for publication 23 Dec. 1988. Scientific Article no. A-4974, Contribution no. 8018 of the Maryland Agricultural Experiment Station (Dept. of Horticulture). The cost of publishing this paper was defrayed in part by the payment of page charges. Under postal regulations, this paper therefore must be hereby marked advertisement solely to indicate this fact.

${ }^{1}$ Associate Professor. or compiled programming language. The Lotus 1-2-3 (L123) worksheet described here, JNTSCALE.WK1, was developed to facilitate these calculations as well as provide estimates of the number of effective factors and of broad and narrow sense heritability using means and variances from six families: two inbred parents, the $F_{1}$ and $F_{2}$, and backcrosses of the $F_{1}$ to each parent

JNTSCALE.WK1 only allows entry of generation means, sample sizes, and variances (as calculated by L123's @VAR function) for each of the six families; all other cells are protected from alteration. As L123 calculates biased estimates of variance (Lotus Dev. Corp., 1987), the worksheet corrects for the bias and also calculates the variance of the mean for the family. For the joint scaling test, the worksheet generates appropriate weights for each family, calculates an information matrix for the three genetic parameters, inverts the $3 \times 3$ information matrix (Tatsuoka, 1971), provides estimates and standard errors for $\mathrm{m}$, [d], and [h], and sums the squares of deviations of observed from expected estimates for each type of family; the residual error sum of squares can then be tested using a $x^{2}$ test.

The six-factor scaling analysis is calculated as described by Mather and Jinks (1982). Estimates for each parameter are calculated and divided by the standard errors of the estimate to test for significance. In addition, the worksheet estimates the number of effective factors controlling the trait (Wright, 1934) and provides an estimate of narrow sense heritability (Warner, 1952) and two estimates of broad sense heritability (Mahmud and Kramer, 1951; Allard, 1960).

This worksheet is of value to breeders and geneticists, since generation means and var- iances can be calculated using L123 and entered into the worksheet. In cases where data transformation is required, recalculation using transformed data can be performed quickly. The worksheet also provides a teaching tool for statistical analysis of genetic variation. Each calculation is referenced, and formulas used for calculating genetic parameters can be examined. Effects of different data sets can be evaluated and results from manual calculations checked. Data distributed with the worksheet are from a wheat heading experiment described by Allard (1960).

JNTSCALE.WKl requires a microcomputer with a spreadsheet program capable of manipulating L123 Rel. 2.1 files (*.WK1). To effectively use the worksheet, users should have a full understanding of the principles of each of these analyses, including knowledge of which conditions are necessary to validate a given test. For instance, calculations for estimating narrow-sense heritability and the number of effective factors assume an absence of dominance and epistasis. Calculations for broad-sense heritability may not be applicable for crops that suffer severe inbreeding depression.

A disk copy (MS-DOS format) of the JNTSCALE.WK1 is available for a nominal charge from me. Alternatively, JTNSCALE.WK1 can be downloaded from HortLine, the ASHS BBS (703/836-2418).

\section{Literature Cited}

Allard, R.W. 1960. Plant breeding. Wiley, New York.

Cavalli, L.L. 1952. An analysis of linkage in quantitative inheritance, p. 135-144. In: E.C.R. Reeve and C.H. Waddington (eds.). Quantitative inheritance. HMSO, London.

Jinks, J.L. and R.M. Jones. 1958. Estimation of the components of heterosis. Genetics 43:223234.

Lotus Development Corporation. 1987. Lotus 12-3 user manual, Release, 2.01. Lotus Dev. Corp., Cambridge, Mass.

Mahmud, I. and H.H. Kramer. 1951. Segregation for yield, height and maturity following a soybean cross Agron. J. 43:605-608.

Mather, K. and J.L. Jinks. 1982. Biometrical genetics, 3rd ed. Chpman and Hall. London.

Tatsuoka, Maurice M: 1971. Multivariate analysis. Wiley, New York.

Warner, J.N. 1952. A method for estimating narrow sense heritability. Agron. J. 44:427-430.

Wright, S. 1934. The results of crosses between inbred strains of guinea pigs, differing in number of digits. Genetics 19:537-551. 